\title{
Fatores de risco para a elevação da pressão arterial em adolescentes
}

\section{Risk factors for high blood pressure in adolescents}

\author{
Laura S. Fonseca', Vanessa R. Kirsten²
}

\begin{abstract}
RESUMO
Modelo do estudo: Estudo transversal. Objetivo: O objetivo deste trabalho foi verificar fatores de risco para elevação da pressão arterial em adolescentes. Métodos: A amostra foi constituída por adolescentes de ambos os sexos com a idade entre 14 e 18 anos. Os fatores de risco foram avaliados pelos dados demográficos, consumo de lanche na escola, prática de atividade física, uso de tabaco e consumo de álcool. Realizou-se avaliação nutricional através do peso e altura e circunferência da cintura, além da verificação da pressão arterial. Resultados: Observou-se que $21.5 \%(n=26)$ dos adolescentes estavam acima do peso, 89.3\% $(n=108)$ estavam dentro dos parâmetros considerados normais para a circunferência da cintura. Observou-se que 26.4\% ( $n=32)$ apresentavam valores limítrofes e 19\% $(n=23)$ estavam com a pressão arterial elevada. Os adolescentes com maior circunferência da cintura tiveram maiores valores de pressão arterial $(P=0.034)$. Conclusão: Este estudo demonstrou que alta prevalência de pressão arterial elevada e a circunferência de cintura pode ser um bom parâmetro para se determinar risco de desenvolver hipertensão arterial, concomitantemente com o estado nutricional.
\end{abstract}

Palavras-chave: Hipertensão. Adolescente. Nutrição do Adolescente. Fatores de Risco. Hábitos Alimentares.

\section{Introdução}

As doenças cardiovasculares são consideradas um importante problema de saúde global, com maior prevalência em paises em desenvolvimento. ${ }^{1}$ Constituem a principal causa de morbimortalidade na população ocidental, no Brasil são responsáveis por mais de $1 / 3$ dos óbitos. ${ }^{2,3}$

A hipertensão arterial sistêmica é uma doença clínica com etiologia multifatorial, proveniente de di- ferentes mecanismos, e é considerada um dos mais importantes fatores de risco para o desenvolvimento de doenças cardiovasculares, explicando $40 \%$ das mortes por acidente vascular encefálico e $25 \%$ daquelas por doença arterial coronariana. ${ }^{4}$

Inquéritos de base populacional realizados em algumas cidades do Brasil apontam altas taxas de hipertensão na população urbana adulta, com valores entre 22.3 a $43.9 \%$. $^{2}$ Ainda que, predomine em adultos, a prevalência de hipertensão arterial em crianças
${ }^{1}$ Acadêmica do Curso de Nutrição

${ }^{2}$ Professora do Curso de Nutrição. Centro Universitário Franciscano - UNIFRA. Área das Ciências da Saúde - Curso de Nutrição
Correspondência:

Prof ${ }^{a}$ Vanessa Ramos Kirsten Centro Universitário Franciscano - Curso de Nutrição Rua dos Andradas, 1614. Centro Telefone: (55) 322012-00 ou (55) 9993-9431

E-mail: vanessark@unifra.br

Artigo recebido em 30/06/2009 Aprovado para publicação em 20/04/2010 
e adolescentes é considerável, tem variação de $2 \%$ a $13 \%$ em diferentes regiões do mundo. No Brasil é demonstrada por alguns estudos epidemiológicos, que apontam taxas entre 6 e $8 \%$ da população jovem ${ }^{5,6}$. Algumas pesquisas internacionais também demonstram um grande número de jovens com a pressão arterial elevada como, o estudo realizado em escolas de Houston nos Estados Unidos com 6790 adolescentes entre 11 e 17 anos, que apresentou prevalência de $15,7 \%$ de pré-hipertensos e 3,2 de hipertensos e, desses, $80 \%$ se encontrava no primeiro estágio. ${ }^{10}$

A hipertensão arterial tem ocorrência devido a uma combinação de múltiplos fatores predisponentes, está associada ao excesso de peso, sedentarismo, dieta inadequada, entre outros. Assim como em adultos, adolescentes com esta enfermidade estabelecida desenvolvem lesão de órgãos alvos, incluindo hipertrofia ventricular esquerda. ${ }^{7,8,9}$

É preocupante o aumento de crianças e adolescentes com predisposição para hipertensão arterial, tendo em vista que os problemas decorrentes desses fatores de risco poderão se associar e causar o desenvolvimento de doenças cardiovasculares precocemente. ${ }^{11} \mathrm{O}$ estilo de vida, hábitos alimentares e antecedentes familiares da doença são alguns fatores que, associados, podem ser preditores da hipertensão arterial na vida adulta. ${ }^{12}$

Desta forma, é de extrema importância a identificação desses fatores de risco e a sua relação com valores pressóricos elevados para minimizar ou prevenir complicações crônicas que podem ser expressas posteriormente. Além disso, a obtenção e publicação de dados sobre pressão arterial sistêmica elevada em adolescentes na região centro do Estado do Rio Grande do Sul são inéditas. O objetivo do presente estudo foi verificar alguns fatores que possam estar implicados no risco de elevação da pressão arterial em adolescentes de uma escola estadual do centro do estado do Rio Grande do Sul.

\section{Materiais e Métodos}

Caracterizado por um estudo transversal, a presente pesquisa foi realizada com adolescentes de uma escola estadual de Santa Maria - RS (Brasil), escolhida aleatoriamente, no período de agosto a setembro de 2008. A amostra foi constituída por adolescentes de ambos os sexos, estudantes do ensino fundamental e médio com a idade entre 14 e 18 anos. Levando em consideração a estimativa do Consenso Brasileiro de
Hipertensão Arterial ${ }^{6}$, que a prevalência de hipertensão arterial é de $8 \%$ em adolescentes e uma precisão desejada de $95 \%$, o cálculo da amostra para um estudo de prevalência ficou em torno de 120 adolescentes estudados. ${ }^{13}$ Realizou-se a apresentação do trabalho nas salas de aula, explicando a metodologia e os objetivos do projeto, enfatizando a importância de sua realização e participação dos alunos. Os adolescentes levaram para casa os termos de consentimento livre e esclarecido (TCLE) para assinatura dos responsáveis que os autorizassem a participar da pesquisa.

Os adolescentes incluídos no estudo foram aqueles em que os pais ou responsáveis expressaram seu consentimento para participação da pesquisa. Foram excluídos do estudo aqueles que recusaram participar, que se encontraram ausentes no dia da aplicação do trabalho ou que apresentassem doenças crônicas diagnosticadas.

A coleta de dados foi realizada através da aplicação de um questionário não validado, contendo perguntas fechadas sobre dados demográficos, o consumo de lanche na escola, prática de atividade física, uso de tabaco e consumo de álcool.

Realizou-se avaliação nutricional através do peso e altura, o primeiro com uma balança digital portátil da marca Techline, com capacidade de $136 \mathrm{~kg}$ com graduação de 100 em 100 gramas situadas em superfície plana e o segundo com uma fita métrica inextensível fixada na parede plana e sem rodapé com auxílio de um esquadro para a localização exata da medida da fita. Os participantes estavam descalços e com o mínimo de roupa possível. O estado nutricional foi classificado pelo Índice de Massa Corporal (IMC) para a idade com classificação do Ministério da Saúde do Brasil. ${ }^{14}$

A circunferência da cintura foi obtida entre o gradil costal e a crista ilíaca superior. Considerou-se a média de três aferições consecutivas como estimativa e foi classificado de acordo com os critérios propostos por Fernández. ${ }^{15}$

A verificação da pressão arterial foi mensurada através de um esfigmanômetro de marca Heijdi e um estetoscópio Rappaport Premium realizada por uma acadêmica de enfermagem. A medida da pressão arterial na posição sentada foi realizada de acordo com os procedimentos descritos: repouso de pelo menos 5 minutos em ambiente calmo, não praticar exercícios físicos 60 a 90 minutos antes, não ingerir bebidas alcoólicas, café ou alimentos e não fumar 30 minutos antes, manter pernas descruzadas, pés apoiados no 
chão, dorso recostado na cadeira e relaxado. Após a primeira verificação, realizou-se um intervalo de 1 a 2 minutos para realizar a medida novamente. $\mathrm{O}$ diagnóstico foi de acordo com os valores referentes aos percentis $90^{\circ}, 95^{\circ}$ e $99^{\circ}$ de pressão arterial para adolescentes, de acordo com idade, sexo e percentis de estatura do avaliado. A classificação para valores abaixo do percentil $90^{\circ}$ é considerada normal; valores entre o percentil $90^{\circ}$ a $95^{\circ}$, limítrofe; valores iguais ou acima a pressão arterial está alterada. ${ }^{5}$ Para análise dos dados, foram utilizados como pressão alterada, os adolescentes classificados como pressão arterial limítrofe e alterada.

A pesquisa foi submetida e aprovada pelo Comitê de Ética em Pesquisa do Centro Universitário Franciscano sob o número do protocolo (registro na Conep $n^{\circ} 1246$ e registro no CEP da UNIFRA: 148.2008.2).

\section{Análise estatística}

Os dados foram analisados através de estatística descritiva simples (média, desvio padrão e porcentagem). Para verificar a comparação entre o estado nutricional e fatores de risco com hipertensão arterial sistêmica, utilizou-se o teste qui-quadrado $\left(X^{2}\right)$ e o teste $T$ de Student quando as variáveis contínuas foram comparadas entre os gêneros. Foi utilizado o software SPSS versão 15.0, e foram consideradas diferenças estatisticamente significativas quando o valor $\mathrm{P}<0.05$.

\section{Resultados}

Dos 164 alunos matriculados, foram avaliados 121 adolescentes, em que, $44.6 \%(n=54)$ eram do sexo masculino e $55.4 \%$ ( $n=67)$ do sexo feminino, e apresentavam média de idade de $15.9 \pm 1.1$ anos variando entre 14 e 18 anos. Em relação ao estado nutricional, observou-se que, $73.6 \%(\mathrm{n}=89)$ dos adolescentes estavam eutróficos e $21.5 \%(n=26)$ estavam acima do peso.

Quanto à circunferência da cintura $89.3 \%$ $(\mathrm{n}=108)$ estavam dentro dos parâmetros considerados normais e, 76,9\% ( $\mathrm{n}=10)$ dos que estavam com a circunferência da cintura aumentada, apresentavam pressão arterial elevada. Observou-se que $26.4 \%$ $(n=32)$ apresentavam valores limítrofes e 19\% ( $n=23)$ estavam com a pressão arterial elevada.

Ao comparar o estado nutricional com a pressão arterial elevada, foi observado que não houve diferença estatisticamente significativa do estado nutricional com os valores de pressão arterial $(\mathrm{P}=0.26)$. No entanto, quando realizada a comparação entre circunferência da cintura aumentada e os valores de pressão arterial, observou-se que os adolescentes que apresentavam maior acúmulo de gordura na região abdominal, pela medida da circunferência da cintura, apresentaram valores mais altos de pressão que os com circunferência da cintura normal. Esta diferença foi estatisticamente significativa $(\mathrm{P}=0.034)$ (Tabela 1$)$.

\section{Tabela 1}

Comparação entre o estado nutricional e circunferência da cintura com pressão arterial em adolescentes, Santa Maria (RS), 2008

\begin{tabular}{lcccc}
\hline & \multicolumn{2}{c}{ Classificação PA } & & \\
& $\begin{array}{c}\text { Normal } \\
\%(n)\end{array}$ & $\begin{array}{c}\text { PA alterada } \\
\%(n)\end{array}$ & $\begin{array}{c}\text { Total } \\
\%(n)\end{array}$ & 0.26 \\
\hline Estado Nutricional & & & & \\
Baixo Peso & $83.3(5)$ & $16.7(1)$ & $100(6)$ & \\
Eutrofia & $56.1(50)$ & $43.9(39)$ & $100(89)$ & \\
Excesso de peso & $47.4(9)$ & $52.6(10)$ & $100(19)$ & \\
Obesidade & $28.6(2)$ & $71.4(5)$ & & \\
Circunferência da Cintura & & & $100(108)$ & \\
Adequada & $58.3(63)$ & $41.7(45)$ & $100(13)$ & \\
Aumentada & $23.1(3)$ & $76.9(10)$ & & \\
\hline
\end{tabular}

Qui-Quadrado $\left(X^{2}\right)$

PA: pressão arterial; \%: porcentagem; n: número de adolescentes. 
Houve diferença estatisticamente significativa quando foi feita a comparação entre o sexo masculino e o feminino quanto ao peso $(\mathrm{P}=0.001)$, circunferência da cintura $(\mathrm{P}=0.009)$ e pressão sistólica $(\mathrm{P}=0.002)$ e diastólica $(\mathrm{P}=0.034)$. Foram observados, com exceção do IMC, valores maiores no sexo masculino para todas as variáveis (Tabela 2).

A Tabela 3 apresenta a comparação dos fatores de risco com a classificação da pressão arterial.
Não houve diferença estaticamente significativa nestas comparações. Porém, observa-se um número maior de adolescentes com a pressão arterial elevada (66.7\%) entre o grupo dos fumantes $(n=6)$.

Ao analisar o tipo de lanche consumido com maior frequiência pelos adolescentes na escola, verificou-se que os mais consumidos são refrigerante (55.4\%), cachorro quente/hambúrguer $(48.8 \%)$, bolacha recheada $(41.3 \%)$, guloseimas $(35.5 \%)$ e o café

\section{Tabela 2}

Comparação entre sexo feminino e masculino de características antropométricas e valores de pressão arterial em adolescentes, Santa Maria (RS), 2008

\begin{tabular}{lrrr}
\hline & Sexo & & \\
& Feminino & Masculino & P* \\
& Média $\pm \mathrm{DP}$ & $65.58 \pm 15.5$ & 0.001 \\
\hline Peso $(\mathrm{Kg})$ & $57.33 \pm 11.7$ & $22.2 \pm 4.3$ & 0.958 \\
$\mathrm{IMC}\left(\mathrm{kg} / \mathrm{m}^{2}\right)$ & $22.3 \pm 4.4$ & $73.87 \pm 10.6$ & 0.009 \\
$\mathrm{CC}(\mathrm{cm})$ & $69.25 \pm 8.5$ & $111.7 \pm 12.1$ & 0.002 \\
PAS $(\mathrm{mmHg})$ & $111.64 \pm 8.6$ & $76.85 \pm 9.7$ & 0.034 \\
PAD $(\mathrm{mmHg})$ & $73.43 \pm 7.9$ & & \\
\hline
\end{tabular}

* Teste t de Student

DP: Desvio-padrão; IMC: Índice de Massa Corporal; CC: Circunferência da Cintura; PAS: Pressão Arterial Sistêmica, PAD: Pressão Arterial Diastólica.

\section{Tabela 3}

Comparação de fatores de risco e pressão arterial em adolescentes, Santa Maria (RS), 2008

\begin{tabular}{|c|c|c|c|c|}
\hline \multirow[b]{2}{*}{ Fatores } & \multicolumn{2}{|c|}{ Classificação PA } & \multirow[b]{2}{*}{$\begin{array}{l}\text { Total } \\
\%(n)\end{array}$} & \multirow[b]{2}{*}{$\mathbf{P} *$} \\
\hline & $\begin{array}{l}\text { Normal } \\
\%(n)\end{array}$ & $\begin{array}{r}\text { PA alterada } \\
\%(n)\end{array}$ & & \\
\hline Raça & & & & 0.75 \\
\hline Branca & $56.8(42)$ & $43.2(32)$ & $100(74)$ & \\
\hline Parda & $54.2(13)$ & $45.8(11)$ & $100(24)$ & \\
\hline Negra & $47.8(11)$ & $52.2(12)$ & $100(23)$ & \\
\hline Atividade física & & & & 0.31 \\
\hline Praticante & $57.1(52)$ & $42.9(40)$ & $100(31)$ & \\
\hline Não praticante & $46.7(14)$ & $53.3(16)$ & $100(90)$ & \\
\hline Fumante & & & & 0.18 \\
\hline Fumante & $33.3(3)$ & $66.7(6)$ & $100(9)$ & \\
\hline Não-fumante & $56.2(63)$ & $43.8(49)$ & $100(112)$ & \\
\hline Álcool & & & & 0.26 \\
\hline Faz uso & $47.6(20)$ & $52.4(22)$ & $100(42)$ & \\
\hline Não faz uso & $58.2(46)$ & $41.8(33)$ & $100(79)$ & \\
\hline
\end{tabular}

* Qui-Quadrado $\left(X^{2}\right)$

PA: pressão arterial; \%: porcentagem; n: número de adolescentes. 
preto (35.5\%). A Tabela 4 demonstra que $58.3 \%$ $(n=25)$ dos adolescentes com pressão arterial elevada têm o hábito de consumir café preto $(\mathrm{P}=0.04)$, e que $61.3 \%(n=38)$ dos adolescentes que não costumavam ingerir cachorro quente/hambúrguer apresentavam pressão arterial normal.

\section{Discussão}

A adolescência é uma fase que há mudanças físicas e psicossociais que acontecem de forma acelerada, o que pode favorecer o desenvolvimento de fatores de risco podendo determinar um perfil cardiovascular desfavorável para esses jovens. Estudos em variadas regiões do Brasil, apontaram a crescente presença de sobrepeso e obesidade, e algumas doenças, como hipercolesterolemia e hipertensão arterial, já na adolescência ${ }^{16}$.

No presente estudo, a prevalência de pressão arterial elevada (19\%) foi superior aos encontrados em pesquisas nacionais, como em Maceió (9.41\%) e no Rio Grande do Sul (6.6\% pressão diastólica elevada, $12.9 \%$ sistólica) ${ }^{3,17}$.

Um dos indicadores para determinar risco de desenvolver hipertensão arterial na vida adulta é o estado nutricional. Vários estudos apontam que a pressão arterial elevada em adolescentes está relacionada ao excesso de peso ${ }^{18-21}$. Um dos motivos dessa relação pode ser atribuído à formação de ateroma na parede dos vasos, na qual resultou de uma maior quantidade de lipoproteínas no sangue ${ }^{22}$.

$\mathrm{O}$ excesso de ganho de peso, principalmente ao final da adolescência, é um importante dado que a Organização Mundial de Saúde (OMS) apresentou recentemente, apontando que, no mundo cerca de $20 \%$ dos adolescentes nessa circunstância apresentam fatores de risco de doenças cardiovasculares ${ }^{16,23}$.

É importante lembrar que alguns adolescentes com excesso de peso já apresentam algum grau de resistência à insulina, e como a pressão arterial elevada, são também componentes da Síndrome Metabólica, esta é caracterizada pela associação de fatores de risco para as doenças cardiovasculares. Antigamente, a hipertensão arterial na infância era considerada um fator de risco independente para essas patologias, mas sua ligação com outros fatores de risco da Síndrome Metabólica requer maior atenção por intensificar o risco de desenvolver doenças cardiovasculares ${ }^{24,25}$

Uma pesquisa realizada por Rosa et al. ${ }^{26}$, com adolescentes com idade entre 12 e 17 anos de escolas públicas da cidade de Niterói - RJ apontou diferença estatisticamente significativa entre a hipertensão e valores acima dos considerados normais para o IMC e circunferência da cintura.

\section{Tabela 4}

Relação entre consumo de lanches e pressão arterial em adolescentes, Santa Maria (RS), 2008

\begin{tabular}{|c|c|c|c|c|}
\hline \multirow[b]{2}{*}{ Alimento/Bebida } & \multicolumn{2}{|c|}{ Classificação PA } & \multirow[b]{2}{*}{$\begin{array}{l}\text { Total } \\
\%(n)\end{array}$} & \multirow[b]{2}{*}{$p$} \\
\hline & $\begin{array}{c}\text { Normal } \\
\%(n)\end{array}$ & $\begin{array}{c}\text { PA alterada } \\
\%(n)\end{array}$ & & \\
\hline Chimarrão & & & & 0.75 \\
\hline Não consome & $54.5(60)$ & $45.5(50)$ & $100(110)$ & \\
\hline Consome & $54.5(6)$ & $45.5(5)$ & $100(11)$ & \\
\hline Café Preto & & & & 0.04 \\
\hline Não consome & $61.6(48)$ & $38.4(30)$ & $100(78)$ & \\
\hline Consome & $41.7(18)$ & $58.3(25)$ & $100(43)$ & \\
\hline Refrigerante & & & & 0.71 \\
\hline Não consome & $53.7(29)$ & $46.3(25)$ & $100(54)$ & \\
\hline Consome & $55.2(37)$ & $44.8(30)$ & $100(67)$ & \\
\hline Cachorro quente/Hambúrguer & & & & 0.16 \\
\hline Não consome & $61.3(38)$ & $38.7(24)$ & $100(62)$ & \\
\hline Consome & $47.5(28)$ & $52.5(31)$ & $100(59)$ & \\
\hline
\end{tabular}

* Qui-Quadrado $\left(X^{2}\right)$

PA: pressão arterial; \%: porcentagem; n: número de adolescentes. 
Embora não tenha ocorrido diferença estatisticamente significativa entre os adolescentes com a pressão arterial normal e alterada para o estado nutricional (IMC), observou-se neste estudo que a maioria dos adolescentes obesos apresenta pressão arterial elevada (71.4\%).

Em uma pesquisa realizada por Araújo et al. em Fortaleza ${ }^{27}$ em uma escola de ensino fundamental e médio com 385 crianças e adolescentes com idade entre 6 e 18 anos mostrou que há uma correlação positiva estatisticamente significativa com a pressão arterial elevada e circunferência da cintura acima dos valores normais para a idade. Este resultado é semelhante ao presente trabalho, em que $76.9 \%$ dos adolescentes que estavam com circunferência aumentada apresentavam níveis pressóricos elevados.

A medida da circunferência da cintura é considerada, atualmente, um bom parâmetro para verificar a associação entre doenças cardiovasculares e obesidade, e está diretamente relacionada ao acúmulo de gordura abdominal ocasionando danos à saúde, tais como redução da lipoproteína de alta densidade, aumento dos níveis de triglicerídeos e ácidos graxos livres circulantes, hiperinsulinemia e resistência à insulina ${ }^{28}$.

Pesquisas recentes apontam uma maior proporção de pressão arterial elevada em meninos ${ }^{29,30}$, corroborando com os dados do presente trabalho. Esta diferença pode estar associada a alterações biológicas que ocorrem neste período, como a maturação sexual, composição corporal, como também a produção de hormônios. ${ }^{31}$ No entanto, há divergências quando se trata de diferenças da pressão arterial entre os sexos mostrada por outros estudos, como o realizado por Ribeiro et al. ${ }^{32}$ em Belo Horizonte com 1450 estudantes que não encontrou diferença entre os gêneros. Levando em consideração que não há uma comprovação científica sobre a maior prevalência de pressão arterial elevada em adolescentes do sexo masculino em relação aos do sexo feminino.

Há dados insuficientes que definem o papel da raça na hipertensão durante a infância e adolescência. Em uma pesquisa realizada com 5.102 crianças e adolescentes, relacionando os principais grupos étnicos com a pressão arterial, concluiu que a raça não é um fator independente de risco para o desenvolvimento de hipertensão arterial $^{33}$. Embora os resultados de outros estudos mostrem que os indivíduos da raça negra têm uma pressão sanguínea mais elevada do que os indivíduos brancos ${ }^{34}$, neste estudo não houve relação entre raça e pressão arterial.

Em relação à atividade física, não houve resul- tados significativos comparando ativos e sedentários, o mesmo foi encontrado em outros estudos. Como a pesquisa realizada na cidade de João Pessoa - PB $^{31}$ com adolescentes de escolas públicas e privadas que constatou que $7.6 \%$ dos adolescentes não ativos tinham a pressão arterial alterada, enquanto $7 \%$ dos ativos também apresentavam alteração na pressão arterial.

No entanto, muitas pesquisas mostram a relação inversamente proporcional entre a atividade física e a pressão arterial elevada. Guedes et al. ${ }^{30}$ realizaram uma pesquisa com 452 adolescentes com idades entre 15 e 18 anos que teve como resultado que $80 \%$ dos participantes que tinham uma prática insuficiente de atividade física apresentavam alterações na pressão arterial, principalmente na sistólica. Estas divergências entre os estudos podem ocorrer devido aos tipos distintos de instrumentos utilizados, que determinam se o adolescente é ativo ou não ativo.

Ao comparar o uso de fumo e álcool com a elevação da pressão arterial, neste estudo, não houve diferença significativa, o que também foi observado em outras pesquisas ${ }^{17,27}$. No entanto, deve-se considerar que há um maior número de adolescentes fumantes com pressão arterial elevada, e que, o viés de informação pode ter ocorrido com a omissão de algumas respostas em relação ao hábito de fumar. No entanto, este fator deve ser levado em consideração, pois segundo uma pesquisa realizada nos Estados Unidos, aproximadamente 3.000 indivíduos fumam pela primeira vez na adolescência, onde a média da idade entre meninos e meninas é de 11.05 anos. ${ }^{35}$

Em um estudo realizado por Pierine em Botucatu - $\mathrm{SP}^{36}$ com indivíduos com a faixa etária entre 6 e 18 anos, foi apontado que o lanche escolar é inadequado em termos de qualidade nutricional, e mostrou diminuída quantidade de fibras, cálcio e ferro, e aumentada quantidade de carboidratos processados, lipídios e sódio. Os alimentos mais consumidos pelos alunos neste estudo, foram doces, bolacha recheada, frios, pães, refrigerantes, batata frita ou chips e salgados. O que também foi verificado no presente estudo, onde se observou um predomínio da ingestão de alimentos gordurosos e ricos em carboidratos, em relação a alimentos mais saudáveis, como leite (14.9\%) e frutas $(10.7 \%)$. Estes dados são preocupantes, pois demonstram um hábito alimentar inadequado.

Estudos epidemiológicos evidenciam que uma alimentação com teor moderado de sódio e gordura juntamente com o consumo adequado de potássio, cálcio, magnésio, ácido fólico, flavonóides e vitamina 
C podem ter a capacidade de influenciar na diminuição dos níveis de pressão arterial ${ }^{7}$.

A cafeína também pode estar relacionada com a elevação da pressão arterial. Um estudo com 159 jovens norte-americanos demonstrou que o elevado consumo de cafeína pelos adolescentes pode alterar a pressão arterial e contribuir para o aumento no risco de hipertensão. ${ }^{37}$ Neste trabalho, $58.3 \%$ dos adolescentes que tem por hábito ingerir café preto frequentemente apresentavam pressão arterial elevada.

Este estudo apresenta limitações metodológicas que devem ser levadas em conta na interpretação de seus resultados, como:

- Para determinar o diagnóstico de hipertensão arterial em adolescentes são necessárias três medidas de pressão arterial em ocasiões diferentes, pois esta é variável ao longo do tempo. O presente estudo não avaliou a hipertensão arterial e sim valores da pressão alterada o que pode justificar valores tão elevados. A literatura recomenda que esta medida possa ser usada como indicador de risco de hipertensão arterial $^{17}$;

- Assim como, não se pode afirmar neste estudo que a relação da circunferência da cintura pode ser um bom parâmetro para se determinar o desenvolvi- mento da hipertensão arterial, no entanto, pode ser um possível risco para a patologia;

- Neste estudo não foi avaliado o processo de maturação sexual e sua influência na pressão arterial, alguns estudos ${ }^{38,39}$ indicam que no período de desenvolvimento dos adolescentes há uma alteração nos níveis pressóricos;

- Utilização de questionários de consumo alimentar e atividade física não validados, podem não apresentar a realidade destas variáveis.

É oportuno destacar a importância da verificação antecipada desses fatores de risco com o intuito de proteção e prevenção de complicações cardiovasculares futuras visto que, na amostra estudada foi observada alta prevalência de pressão arterial alterada.

Medidas educacionais buscando um hábito de vida saudável é um método efetivo para minimizar tais doenças. Manter um estilo de vida saudável, um estado nutricional adequado, por meio de um padrão alimentar equilibrado e a prática de atividade física regular são prioridades para evitar a elevação da pressão arterial e, assim, impedir o aumento de um possível risco para o desenvolvimento de hipertensão arterial na vida adulta ${ }^{40}$.

\begin{abstract}
Model of the study: Transversal study. Objective: The purpose of this study was to verify risk factors for high blood pressure in teenagers. Methods: The sample was consisted of adolescents of both sexes with age between 14 and 18 years. The risk factors were assessed by demographics, consumption of snack at school, physical activity, tobacco use and alcohol consumption. The nutritional assessment was held by height and weight and waist circumference, in addition to checking blood pressure. It was found that $21.5 \%(n=26)$ of adolescents were overweight and it was not related to the values of high blood pressure. Results: About the waist circumference $89.3 \%(n=108)$ were within the parameters considered normal. It was found that $26.4 \%(n=32)$ had borderline values and $19 \%(n=23)$ were classified as hypertensive. The adolescents with greater waist circumference had higher blood pressure $(P=0.034)$. Conclusion: This study showed that the circumference of waist may be a good parameter to determine risk of developing hypertension, concomitant with the nutritional condition. The prevention of obesity throught an appropriated dietary pattern and practice of regular physical activity is a priority for positive impact on the improvement of hypertension, reducing the risk for cardiovascular disease and thereby, increasing life expectancy.
\end{abstract}

Key words: Hypertension. Adolescents. Adolescent Nutrition. Risk Factors. Food Habits.

\section{Referências Bibliográficas}

1- Yusuf S, Reddy S, Ounpuu S, Anand S. Global burden of cardiovascular diseases: part II: variations in cardiovascular disease by specific ethnic groups and geographic regions and prevention strategies. Circulation. 2001; 104: 2855-z8.
2- Mendes MJ, Alves JG, Alves, AV, Siqueira PP, Freire EF. Associação de fatores de risco para doenças cardiovasculares em adolescentes e seus pais. Rev Bras Saúde Matern Infant. 2006; 6 (Supl 1): S49-S54.

3- Mendonça G, Kuschnir M. Risk factors associated with arterial hypertension in adolescents. J Pediatr. 2007; 83: 335-42. 
4- Sociedade Brasileira de Hipertensão. IV Diretrizes Brasileiras de Hipertensão Arterial. Arq Bras Cardiol. 2004; 82(4). Avaiable from: http://www.scielo.br/pdf/abc/v82s4/04.pdf

5- Sociedade Brasileira de Hipertensão. V Diretrizes Brasileiras de Hipertensão Arterial. Arq Bras Cardiol. 2006. Avaiable from: http://www.sbn.org.br/Diretrizes/V_Diretrizes_ Brasileiras_de_Hipertensao_Arterial.pdf

6- Sociedade Brasileira de Hipertensão. III Consenso Brasileiro de Hipertensão arterial. Arq Bras Cardiol. 1999. Available from: http://www.sbn.org.br/Diretrizes/cbha.htm

7- National Heart Lung and Blood Institute. DASH eating plain. United States, 2006. Available from: http://www.nhlbi. nih.gov/health/public/heart/hbp/dash/new_dash.pdf

8- Hanevold C, Waller J, Daniels S, Portman R, Sorof J. The effects of obesity, gender and ethnicity on left ventricular hypertrophy and geometry in hypertensive children a collaborative study of the International Pediatric Hypertension Association. Pediatrics. 2004; 113: 328-33.

9- Daniels SR, Loggie JM, Khoury P, Kimball TR. Left Ventricular Geometry and Severe Left Ventricular Hypertrophy in Children and Adolescents With Essential Hypertension. Circulation. 1998; 97: 1907-11.

10- McNiece KL, Poffenbarger TS, Turner JL, Franco KD, Sorof JM, Portman RJ. Prevalence of hypertension and pre-hypertension among adolescents. J Pediatr. 2007; 150: 640-4.

11- Salgado CM, Carvalhases JT. Hipertensão arterial na infância. J Pediatr. 2003; 79(Supl 1): 115-24.

12- Silva MA et al. Prevalência de Fatores de Risco Cardiovascular em Crianças e Adolescentes da Rede de Ensino da Cidade de Maceió. Arq Bras Cardiol. 2005; 84: 387-92.

13- Pereira MG. Epidemiologia: Teoria e Prática. Capitulo. 6.ed. Rio de Janeiro: Guanabara Koogan; 2002. p. 298-306.

14- Ministério da Saúde. Saúde da Família. Growth reference 519 years, 2007. Available from: http://www.saude.gov.br/ nutricao.php

15- Fernández JR, Reddnen D, Pietrobelli A, Allison D. Waist circumference percentiles in nationally representative samples of African-American, European-American, and Mexican-American children and adolescents. J Pediat. 2004; 145: 439-44.

16- Rego AL, Chiara VL. Nutrição e excesso de massa corporal: fatores de risco cardiovascular em adolescentes. Rev Nutr Camp. 2006; 19:705-12.

17- Moura AA, Silva MA, Ferraz, MR, Riveira IR. Prevalência de pressão arterial elevada em escolares e adolescentes de Maceió. J Pedriat. 2004; 80: 35-40.

18- Sorof JM, Lai D, Turner J, Poffenbarger T, Portman RJ. Overweight, ethnicity, and the prevalence of hypertension in schoolaged children. Pediatrics. 2004; 113: 475-82.

19- Chiolero A, Madeleine G, Gabriel A, Burnier M, Paccaud F, Bovet P. Prevalence of elevated blood pressure and association with overweight in children of a rapidly developing country. J Hum Hypertens. 2007; 21:120-7.

20- Messiah SE, Arheart KL, Lipshultz SE, Miller TL. Body mass index, waist circumference, and cardiovascular risk factors in adolescents. J Pediatr. 2008; 153:845-50.

21- Ostchega Y, Carroll M, Prineas RJ, McDowell MA, Louis T, Tilert T. Trends of elevated blood pressure among children and adolescents: data from the National Health and Nutrition Examination Survey 1988-2006. Am J Hypertens. 2009; 22:59-67.

22- Sinaiko A. Obesity, insulin resistance and the metabolic syndrome. J Pediatr. 2007; 83:3-4.
23- Freitas JB, Tavares A, Kohlmann O, Zanella MT, Riberio, AB. Estudo transversal sobre o controle da pressão arterial no Serviço de Nefrologia da Escola Paulista de Medicina. Arq Bras Cardiol. 2005; 79:387-92.

24- Reaven GM. Insulin resistance/compensatory hyperinsulinemia, essential hypertension, and cardiovascular disease. J Clin Endocrinol Metab. 2003; 88:2399-403.

25- The Four Report on the Diagnosis, Evaluation and Treatment oh High Blood Pressure in Children and Adolescents. Pediatrics. 2005; 114: 555-76.

26- Rosa ML, Mesquita ET, Rocha ER, Fonseca VM. Índice de Massa Corporal e Circunferência da Cintura como Marcadores de Hipertensão Arterial em Adolescentes. Arq Bras Cardiol. 2007; 88:573-8.

27- Araújo TL. Análise de indicadores de risco para hipertensão arterial em crianças e adolescentes. Rev Esc Enferm USP. 2008; 42:120-6.

28- Sarni RS, et al. Relação da cintura abdominal com a condição nutricional, perfil lipídico e pressão arterial em pré-escolares de baixo estrato socioeconômico. Arq Bras Cardiol. 2006; 89:153-8

29- Rosa ML, Fonseca VM, Oigman G, Mesquita ET. Pré-hipertensão arterial e pressão de pulso aumentada em adolescentes: prevalência e fatores associados. Arq Bras Cardiol. 2006; 87:46-53.

30- Guedes DP, Guedes JERP, Barbosa DS, Oliveira JA, Stanganelli LCR. Fatores de risco cardiovasculares em adolescentes: indicadores biológicos e comportamentais. Arq Bras Cardiol. 2006; 86:439-50.

31- Silva KS, Júnior JC. Fatores de risco associados à pressão arterial elevada em adolescentes. Rev Bras Med Esporte. 2007; 13:237-40.

32- Ribeiro RQ, Lotufo PA, Lamounier JA, Oliveira RG, Soares JF, Botter DA. Fatores adicionais de risco cardiovascular associados ao excesso de peso em crianças e adolescentes. Arq Bras Cardiol. 2006; 86:408-18.

33- Sorof JM, Lai MD, Turner J, Poffenbarger T, Portman RJ. Children Overweight, Ethnicity, and the Prevalence of Hypertension in School-Aged. Pediatrics. 2004; 113:475-82.

34- Luma GB, Spiotta R. Hypertension in children and adolescents. American Family Physician. 2006; 73: 1558-68.

35- Barbosa MT, Cotrim CB, Filho AR. O uso de tabaco em estudantes de primeiro e segundo grau de dez capitais brasileiras: possíveis contribuições de estatística multivariada para a compreensão do fenômeno. Rev Saúde Pública. 1989; 23: 401-9.

36- Pierine DT et al.Composição corporal, atividade física e consumo alimentar de alunos do ensino fundamental e médio. Motriz. 2006; 12:113-24.

37- Savoca MR, Evans CD, Wilson ME, Harshfield GA. The association of caffeinated beverages with blood pressure in adolescents. Arch Pediatr Adolesc Med. 2004; 158: 473-7.

38- Cho SD, Mueller WH, Meininger JC, Liehr P, Chan W. Blood pressure and sexual maturity in adolescents: The Heartfelt Study. Am J Hum Biol. 2001;13:227-234.

39- The influence of sexual maturation on blood pressure and body fatness in African-American adolescent girls and boys. Am J Hum Biol. 2009; 21:105-12.

40- Brandão AP, Brandão AA, Berenson GS, Fuster V. Síndrome Metabólica em crianças e adolescentes. Arq Brasil Cardiol. 2005; 85:79-81. 